\title{
Building a Formal Framework for Mobile Ad Hoc Computing
}

\author{
Lu Yan and Jincheng Ni \\ Turku Centre for Computer Science (TUCS) and \\ Department of Computer Science, Åbo Akademi University, \\ FIN-20520 Turku, Finland. \\ $\{$ Lu.Yan, Jincheng.Ni\}@abo.fi
}

\begin{abstract}
We present a formal framework towards a systematic design for MANET applications. In this paper, we define a layered architecture for mobile ad hoc computing and specify the system components with the B method and UML diagrams.
\end{abstract}

\section{Introduction}

We define a layered architecture in Fig. 1 for mobile ad hoc computing and propose a middleware layer with three key components between software application layer and ad hoc networking layer. We specify the system components with the $\mathrm{B}$ method [1], and model the interactions and message communications between components with UML diagrams.

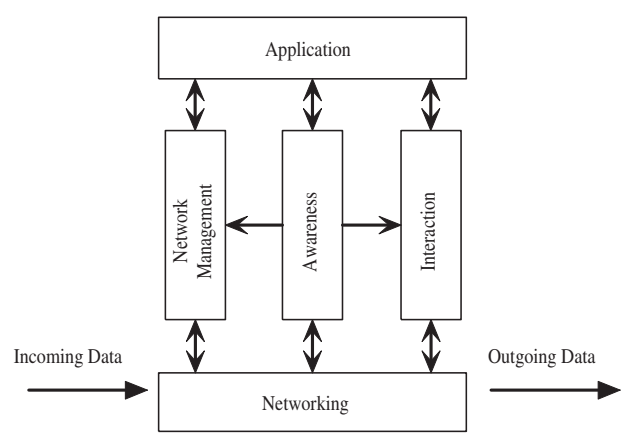

Fig. 1. MANET Architecture

\section{Network Management}

There is no constant topology or centralized manager in MANET. In order to form a self-organizing network, and support multi-hop routing by forwarding packets, it is necessary to have the network management in every node in MANET. 


\section{Awareness}

As shown in Fig. 2 a node processes incoming messages according to the format of data packets. If the received message is a communication message, the system

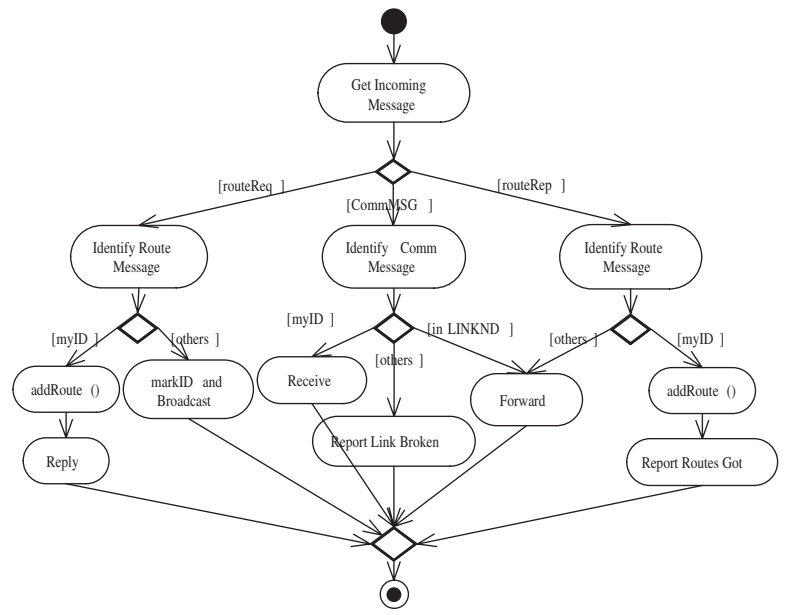

Fig. 2. Incoming Message Processing

checks the packet head, and then receives or forwards the packet according to the next hop ID of the route. In case the ID is unrecognizable, the system will report a broken route. If the incoming message is a routing message, the system will process the message according to the current routing protocols in MANET.

\section{Interaction}

We consider an opening session for the interactive communication between nodes. In such a session, the source and destination nodes exchange messages and update routing information for communication. As shown in Fig. 3 when the system opens such a session and starts interactive communication, the source node will select a route from the routing table or detect a new route to reach the destination node. If there is no available route or the destination node is not detected in the network, the opening session fails and a failure message is sent back to the source node. In a successful case, once a route is available, a communication session between the source node and destination node is created and the interactive communication starts.

During the interactive communication, the network topology might be changed and it might lead to a broken route. Thus route maintenance and recovery are needed for interactive communication. Figure 4 shows how a route is recovered when the system knows that the route is broken. In our design, it is 


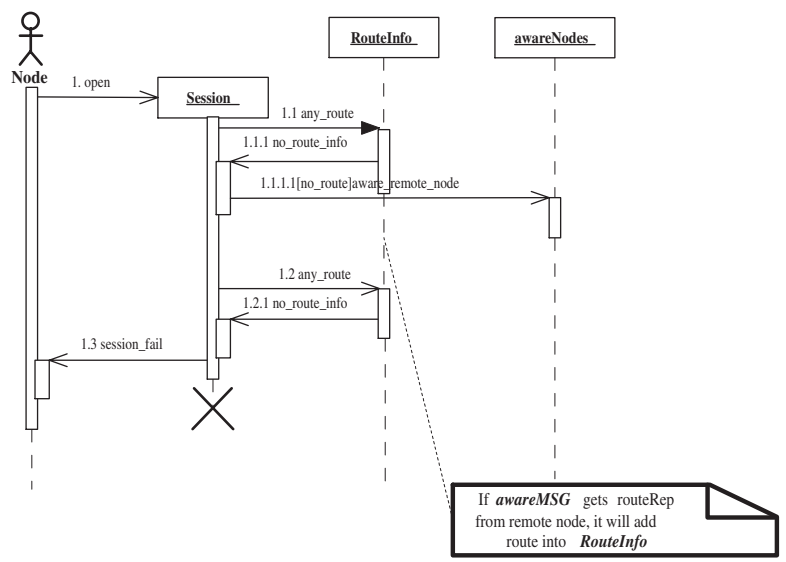

Fig. 3. Opening Session for Interactive Communication

assumed that multiple routes discovery protocols are used. For example, when source node $\mathrm{S}$ is communicating with destination node $\mathrm{D}, \mathrm{S}$ sends data packets to D along with the selected route. During their communication, if $\mathrm{S}$ gets to know that the communication route is broken, $\mathrm{S}$ doesn't need to rediscover a new route immediately because $\mathrm{S}$ might have detected several routes in the previous discovery. It can then choose another available route and replace the broken one. Until all the routes are not reachable to the destination, the system will start route discovery again [2].

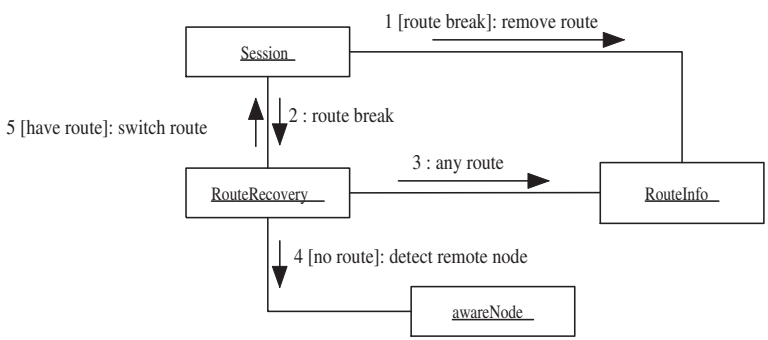

Fig. 4. Route Maintenance and Recovery

\section{Relationship of Components}

There are three components in the system specification, which are built up with nine B machines. Two pre-defined machines AdHocNet and RouteInfo are used to specify the context and environment of mobile ad hoc computing. The component Network Management is composed of three machines: netManager, modeSet 
and Connector, and the component Awareness has two machines: awareNodes and awareMSG. There are two machines: Communication and RouteRecovery in the component Interaction. For the whole system, the relationship of machines within components and between components is shown in Fig. 5 .

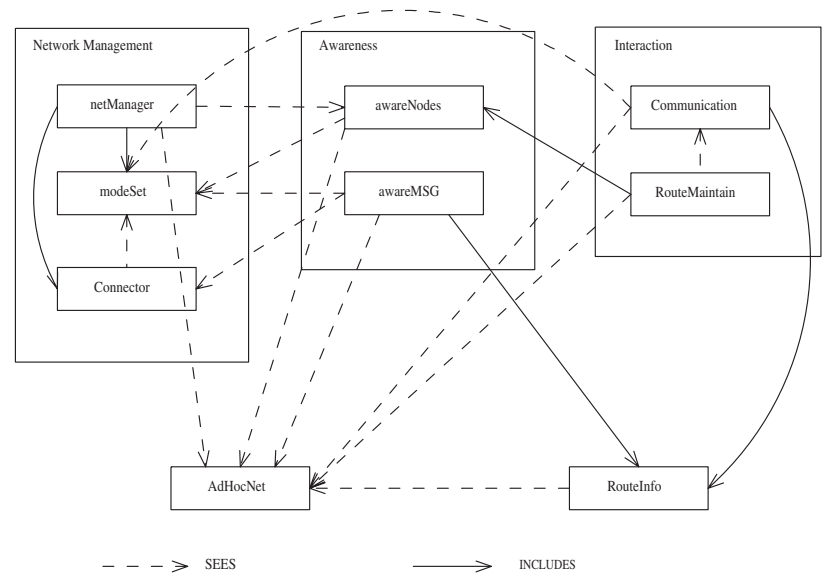

Fig. 5. Relationship of Components

\section{Concluding Remarks}

The goal of the specification is a formal framework to enable applications to be developed based on the three components, which are to be executed arbitrarily in MANET. A complete detailed specification of mobile ad hoc computing and some experiment results can be found at $\underline{3}$.

Acknowledgements. This work was supported by the Mobile Ad Hoc Computing project directed by Kaisa Sere, and we are grateful for Kaisa's contribution.

\section{References}

1. E. Sekerinski and K. Sere (Eds), Program Development by Refinement: Case Studies Using the B Method, Springer-Verlag, 1999.

2. Z. Ye, S. V. Krishnamurthy and S. K. Tripathi, A Framework for Reliable Routing in Mobile Ad Hoc Networks, Proceedings of the IEEE INFOCOM 2003, San Francisco, USA, 2003.

3. L. Yan, J. Ni and K. Sere, Towards a Systematic Design for Ad hoc Network Applications, Proceedings of the 15th Nordic Workshop on Programming Theory (NWPT'03), Turku, Finland, Oct. 2003. 